\title{
Exploration of Residue Binding Energy of Potential Ankyrin for Dengue Virus II from MD Simulations
}

\author{
Wei Lim Chong ${ }^{1 *}$, Sharifuddin M. Zain ${ }^{1}$, Noorsaadah Abd. Rahman ${ }^{1}$, Rozana Othman ${ }^{2}$, Shatrah Binti Othman ${ }^{3}$, \\ Piyarat Nimmanpipug ${ }^{4}$, Chatchai Tayapiwatana ${ }^{5}$, Vannajan Sanghiran Lee $e^{1,4}$ \\ ${ }^{l}$ Department of Chemistry, Faculty of Science, University of Malaya 50603 Lembah Pantai, Kuala Lumpur Malaysia. \\ ${ }^{2}$ Department of Pharmacy, Faculty of Medicine, University of Malaya. 50603 Lembah Pantai, Kuala Lumpur Malaysia. \\ ${ }^{3}$ Department of Molecular Medicine, Faculty of Medicine, University of Malaya. 50603 Lembah Pantai, Kuala Lumpur Malaysia. \\ ${ }^{4}$ Computational Simulation and Modelling Laboratory (CSML), Department of Chemistry and Center of Excellence for Innovation in \\ Chemistry and Materials Science Research Center, Faculty of Science, Chiang Mai University, Chiang Mai 50200, Thailand. \\ ${ }^{5}$ Division of Clinical Immunology, Department of Medical Technology, Faculty of Associated Medical Sciences, Chiang Mai University \\ Chiang Mai 50200,Thailand.
}

Received: 7 October 2014 / Accepted: 30 November 2014

\begin{abstract}
:
Computational approach was employed to evaluate the binding activity of potential ankyrin and domain III of the envelope protein of dengue virus II. Ankyrin serves as an alternative to antibody due to several advantages. Both the ankyrin and domain III were docked using Z-dock protocol in Discovery Studio suite of programme. The docked complex was simulated under GPU-accelerated workstation for long time scale and followed by binding free energy calculation using Molecular Mechanics-Poisson-Boltzmann Surface Area/Generalized Born Solvent Area (MM-PBSA/GBSA). Decomposed binding free energy located the possible hot spots on ankyrin and also domain III. Several amino residues on ankyrin were observed to be potent mutation points by comparing the energy from snapshots extracted during 3 ns and 6 $10 \mathrm{~ns}$.
\end{abstract}

Key words: Ankyrin, domain III, dengue virus, molecular dynamics simulations, MM-PBSA/GBSA, decomposed energy

\section{Introduction}

Dengue viruses (DENV) are flaviviruses which belong to the family of Flaviviridae and is of mosquito-borne human pathogens [1]. Up-to-date, there are four distinct DENV have been documented, DENV1, DENV2, DENV3 and DENV4. The transmission vector of DENV is mosquito Aede saegypti and Aedes albopictus. Proteins of the viruses have been the target in drug design to inhibit the DENV infection. It is therefore extensive studies have been done on the structural proteins and non-structural proteins. Several reports suggested that DENV enters the host hell through endocytosis and medicated by receptor [2-4]. Among the proteins of DENV, envelope (E) protein has been gained interest and being targeted. The $\mathrm{E}$ protein was observed to form trimers and mediates the fusion between virus and membrane in acidic condition [5]. It opens a door for inhibition by blocking the entry of the virus by targeting the $\mathrm{E}$ protein. $\mathrm{E}$ protein is made up of three domains, domain I, II and III. Of the domains, domain III was observed to involve in binding with the host cell [6].

There is an immunoglobulin-like fold which is a common structure as cell receptor and it was observed to project further away from both domains I and domain II [7]. Domain III was found that respond to the antibody neutralisation as well [8-11]. Roehrig and colleagues [12] pointed that the neutralisation sites of domain III of flaviviruses are of important and specific. Furthermore, the most efficient inhibition on the viral attachment was observed when Monoclonal antibody (MAb) bind with the epitopes on the D-III [13]. Crystallographic study from Lok and colleagues [14] identified a total of 11 important residues on D-III od DENV2 which served as a target site.

Compiling the data from the mentioned literatures, it encourages the exploration on D-III for inhibition purpose. MAbs have been given attention on the drug development for neutralizing effects. Generally antibodies are virtually bind to target specifically with high affinity while giving neutralizing effect [15-16]. Nonetheless, formulation and architecture of antibodies are complicated besides low tissue penetration [17]. The mentioned limitations however promote the development on other proteins which can serve as a substitution of antibodies. Ankyrin Repeat (AR) proteins are one of the considerations for antibodies substitution due to their abundance in nature [18]. Each repeat of the AR protein is made up of a total of 33 amino acid which forms the $\beta$-turn and two anti-parallel $\alpha$-helices and a loop connecting the next repeat [19-20]. Each of the repeats was observed to interact with the target [20].

\footnotetext{
*Corresponding author: Wei Lim Chong,

E-mail: wlchongwilliam@gmail.com
} 
The domains of the AR are to mediate protein-protein interactions. Specific DARPins (designed ankyrin repeat proteins) were selected by Steiner and co-workers [21] to against various targets including HER2, reported with subnanomolar affinities. Recently, Sawatree and colleagues [22] were successfully to demonstrate the potential of AR protein served as a novel class of intracellular antivirals. The valuable findings from the researchers mentioned encouraged the development of AR proteins in inhibiting the dengue virus. Computational approach allows the study of the binding free energy, molecular interactions together with the hot spots of the proteins at molecular level. Performing dynamic study by Molecular Dynamics simulation assists us to explore the binding affinity and evaluate the favourable binding sites on both AR protein and D-III. It is necessary to perform simulation prior to experiments in order to picture the protein-protein interactions. The results obtained will enable further mutation on the AR protein in order to enhance the binding.

\section{Experimental}

The potent ankyrin was proposed by aligning the sequence of binding sites of D-III as reported by Lok and colleagues [14], to the ligand bound to ankyrin obtained from PDB. The aligned sequence of ligand of complex $2 \mathrm{DZN}$ was found in the binding region. Therefore the ankyrin was selected as the potential ankyrin. The starting structure of AR and D-III was retrieved from the complex deposited in the Protein Data Bank (PDB), 2DZN and 2R29 respectively. Both of the D-III and ankyrin were docked under Z-docker protocol on Discovery studio programme version 2.5. The CHARMm force field is employed to account for the atomic charges of the proteins. The model with the highest score after refinement (R-dock protocol) concerning short and long range electrostatics and desolvation energies was selected as the best docked pose which would proceed to MD simulations.

The protein complex was added with $\mathrm{Na}^{+}$ions for neutralizing purpose and then solvated in a cubic box by 40149 TIP3P water molecules in AMBER 12 [23] suite of programme prior to $\mathrm{MD}$ simulation. All the $\mathrm{MD}$ simulations were performed using PMEMD.CUDA from AMBER 12 on graphical processors (GPUs) Quadro 2000D manufactured by NVIDIA. Employing GPU would shorten the simulation wall time required to collect the properties from each simulation. Molecular characteristics of the complex were described by ffSB 12 force field parameters.

The MD simulations are performed using a time step of 2 fs. The long range interactions were taken into account using periodic boundary condition based on the Particle Mesh Ewald (PME) method. The SHAKE algorithm and the Langevin dynamics are applied to constrain bond involving hydrogen and to control the temperature, respectively.
Initially, the temperature of each system was increased gradually from 0 to $310 \mathrm{~K}$ over a period of 60 ps of NVT dynamics. This was followed by 300 ps of NPT equilibration at $310.15 \mathrm{~K}$ and 1 atm pressure and then $10,000 \mathrm{ps}$ of NPT-MD simulation was performed for properties collection. The complex was simulated for 10 ns. The binding free energy and analysis of the hot-spot residues of proteins can be achieved using the Molecular Mechanics - Poisson - Boltzmann Surface Area/ Generalized Born Surface Area (MM-PPSA/GBSA) protocols. They are employed for the decomposition energy calculations which allow the exploration of hot spots on the proteins. The complex interaction energy profiles were generated by decomposing the total binding free energies into residue-residue interaction pairs by the MM-GBSA decomposition process in the mm pbsa program of AMBER 12. The calculation was based on 500 snapshots from the respective trajectories as guided by RMSD plot.

\section{Results and Discussion}

Root Mean Squared Deviations (RMSD) plot of all C $\alpha$ atoms (Figure 1) was plotted to evaluate the structural and conformational changes along the simulation. RMSD of the ank-domain III complex was observed to oscillate steadily within the range about $2.3 \AA-3.7 \AA$ along the simulation. Greater fluctuation was observed occur during 4-6 ns while the earlier stage (1-3 ns) and the latter stage (6-10) were rather steady. The results indicated that the complex did not experience a large conformational and structural changes and it was stable due to the RMSD evolved within the acceptable range.

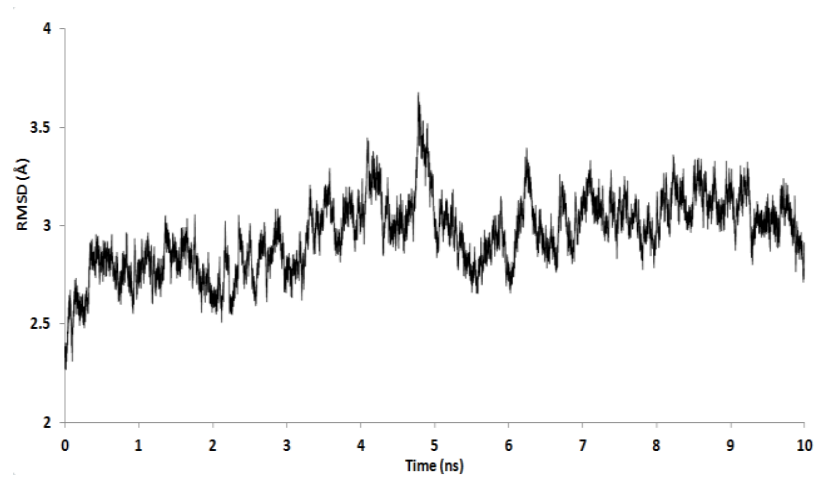

Figure 1. Root Mean Square Deviations (RMSD) of all $\mathrm{C}_{\alpha^{-}}$ atoms of ank-domain III complex.

The energies terms that contributed to the binding free energy were tabulated in Table 1. Evaluation of energies terms was performed using MM-PBSA and MM-GBSA protocols. In this study, PBTOT values were selected for discussion as energies evaluated from MM-GBSA approach were not able to give a good correlation. Comparison was done between the structures of the equilibrium state (3 ns and 6-10 ns) as suggested by RMSD plot as shown in Figure 1. The PBTOT values of complex increased from -28.64 to -24.11 which may imply that the binding activity of ankyrin to domain III 
decrease along the simulation. This was not surprising us as the ankyrin was not specifically designed yet to target the domain III through a strong binding. Despite the binding affinity at the stage was not so high, however, it opened us a door to enhance the binding through mutation.

Table 1. Contribution of energies to binding free energy of ank-domain III complex

\begin{tabular}{|c|c|c|c|}
\hline \multirow{3}{*}{ Method } & \multirow{3}{*}{ Contribution } & \multicolumn{2}{|c|}{$\begin{array}{l}\text { Energy in each contribution } \\
\left(\mathrm{kcal} \mathrm{mol}^{-1}\right)\end{array}$} \\
\hline & & \multicolumn{2}{|c|}{ Ank-Domain III } \\
\hline & & $3 \mathrm{~ns}$ & $6-10 \mathrm{~ns}$ \\
\hline \multirow[t]{4}{*}{ MM } & ELE & -83.55 & -47.37 \\
\hline & VDW & -64.01 & -49.17 \\
\hline & INT & 0 & 0 \\
\hline & GAS & -147.56 & -96.54 \\
\hline \multirow[t]{5}{*}{ PBSA } & $\mathrm{PB}_{\text {SUR }}$ & -13.87 & -10.12 \\
\hline & $\mathrm{PB}_{\mathrm{CAL}}$ & 132.96 & 82.55 \\
\hline & $\mathrm{PB}_{\mathrm{SOL}}$ & 119.10 & 72.43 \\
\hline & $\mathrm{PB}_{\mathrm{ELE}}$ & 49.41 & 35.17 \\
\hline & $\mathrm{PB}_{\mathrm{TOT}}$ & -28.46 & -24.11 \\
\hline \multirow[t]{5}{*}{ GBSA } & $\mathrm{GB}_{\mathrm{SUR}}$ & -6.43 & -4.78 \\
\hline & $\mathrm{GB}_{\mathrm{CAL}}$ & 152.69 & 101.01 \\
\hline & $\mathrm{GB}_{\mathrm{SOL}}$ & 146.26 & 96.23 \\
\hline & $\mathrm{GB}_{\mathrm{ELE}}$ & 69.15 & 53.63 \\
\hline & $\mathrm{GB}_{\mathrm{TOT}}$ & -1.3 & -0.31 \\
\hline
\end{tabular}

Note: ELE: electrostatic interactions, VDW: van der Waals interactions, INT: internal energies, GAS (or MM energies) $=\mathrm{ELE}+\mathrm{VDW}+\mathrm{INT}, \mathrm{PB}_{\mathrm{SUR}}$ : non-polar contribution to solvation, $\mathrm{PB}_{\mathrm{CAL}}$ : polar contribution of solvation, $\mathrm{PB}_{\mathrm{SOL}}=\mathrm{PB}_{\mathrm{SUR}}+\mathrm{PB}_{\mathrm{CAL}}, \mathrm{PB}_{\mathrm{ELE}}=\mathrm{PB}_{\mathrm{CAL}}+\mathrm{ELE}, \mathrm{PB}_{\mathrm{TOT}}$ : total binding free energy calculated by the MM-PBSA method $=\mathrm{PB}_{\mathrm{SOL}}+\mathrm{ELE}$

Examining the relative decomposed binding free energy of the key amino acid residues of ankyrin (Figure 2) provided us information on hot spots of binding in the complex. Note that the amino acid residues of ankyrin from the randomized position of the repeats were evaluated without the concerning the framework residues. Several residues were suggested to be important to contribute in binding due to a large relative energy $(>1$ $\mathrm{kcal} \mathrm{mol}^{-1}$ ) for stabilization purpose. Several residues (Arg36, Trp72, Val107, Ser 148 and Val149) of AR 1SVX (Figure 3) were verified to have significant effective contributions to the relative energy larger than $1 \mathrm{kcal} \mathrm{mol}^{-1}$ for the stabilization energy. On the other hand, energy increase was observed at several residues, Arg36, Val 107 and Val149. These residues could be further investigated by mutation to understand how mutation giving an impact on the binding affinity either enhance or harm the binding activity.

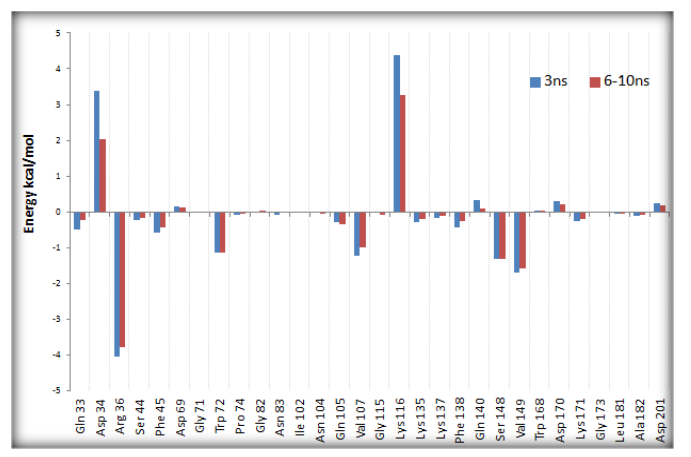

Figure 2. Relative decomposed free energies during 3 ns and 6-10 ns of important amino acid residue of ankyrin.

Three residues of the binding sites as reported by Lok [14] were further studied by Gromowski [7]. Mutation of these three residues harmed the binding of antibody and domain III. This implied that these three residues could be crucial binding. From Figure 3, only residue Ile 312 exhibited a negative energy while the rest exhibited a positive energy. Residue Lys 310 is conserved among the four DENVs and it showed a decrease of energy during 6-10 ns. It suggested that the ankyrin could probably interact with domain III. Therefore, further mutation might able to enhance the binding.

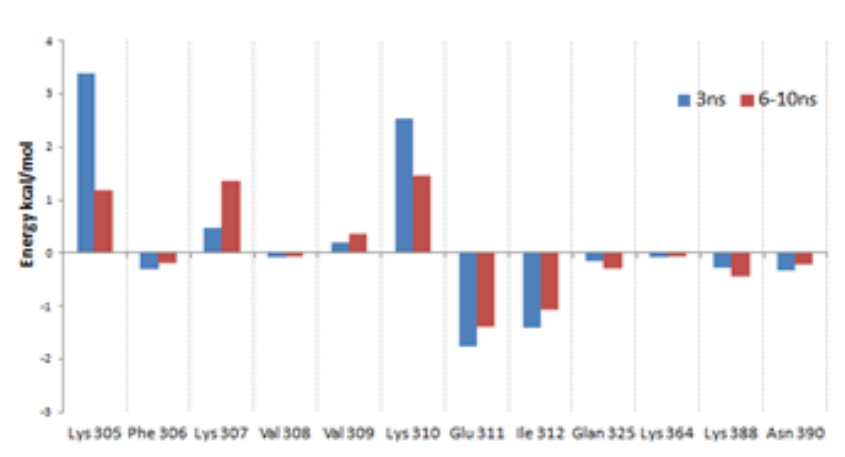

Figure 3. Relative binding free energies during 3 ns and 6-10 ns of important amino acid residue of domain III.

\section{Conclusions}

We have explored the binding activity of ankyrin and domain III of dengue virus type II. Potential ankyrin was obtained from sequence alignment whereas binding sites of domain III was obtained from antibody-domain III complex retrieved from protein data bank. Long time scale simulation under GPU-enabled workstation speeded up the time required for collecting the molecular properties. MM-PBSA and MM-GBSA approaches were employed to account for the binding free energy and it was further decomposed to pair-wise residue energy. Several residues on ankyrin were identified to be the mutation points for enhancing the binding. Decomposed free energy of binding sites of domain III proposed that the selected ankyrin is capable to interact with domain III. All the crucial residues on ankyrin and domian III required further evaluation in order to overview the binding affinity of ankyrin towards domain III.

\section{Acknowledgement}

This research is conducted with the support of University Malaya Research Grant (UMRG-Project No. RP001C13ICT), Computation \& Informatics $(\mathrm{C}+\mathrm{i})$ Research Cluster/High Performance Scientific Computing Program and UMBIO Research Cluster (UMRG-Project No. RP002-2012D) and Fundamental Research Grant Scheme (FRGS-Project No. FP002-2012A and FP0132013A). 


\section{References}

[1] J. Lescar, D. Luo, T. Xu, A. Sampath, S. P. Lim, B. Canard and S. G. Vasudevan, Towards the design of antiviral inhibitors against flaviviruses: the case for the multifunctional NS3 protein from Dengue virus as a target, Antiviral Res., 80(2), 2008, 94-101.

[2] B.D. Lindenbach, H.J. Thiel, and C.M. Rice, "Flaviviridae: the viruses and their replication, In: Knipe DM, Howley PM, eds. Fields virology, 5th ed. Philadelphia: Lippincott William \& Wilkins, 2007, 1101-1152.

[3] F.X. Heinz and K. Stiasny, Flavivirus membrane fusion, $J$. Gen. Virol., 87(10), 2006, 2755-2766.

[4] V.B. Randolph and V. Stollar, Low pH-induced cell fusion in flavivirus-infected Aedes albopictus cell cultures, J. Gen. Virol, 71, 1990, 1845-1850.

[5] S.L. Allison, J. Schalich, K. Stiasny, C. W. Mandl, C. Kunz, and F.X. Heinz, Oligomeric rearrangement of tick-borne encephalitis virus envelope proteins induced by an acidic $\mathrm{pH}$, J. Virol., 69(2), 1995, 695-700.

[6] F.A. Rey, F.X. Heinz, C. Mandl, C. Kunz, and S.C. Harrison, The envelope glycoprotein from tick-borne encephalitis virus at 2 A resolution, Nature, 375(6529), 1995, 291-298.

[7] G.D. Gomowski, J.T. Roehrig, M.S. Diamond, J.C Lee, T.J. Pitcher, and A.D. Barret, Mutations of an antibody binding energy hot spot on domain III of the dengue 2 envelope glycoprotein exploited for neutralization escape, J. Virol., 407(2), 2010, 237-246.

[8] G.D. Gromowski, N.D. Barrett, and A.D. Barrett, Characterization of dengue virus complex-specific neutralizing epitopes on envelope protein domain III of dengue 2 virus, J. Virol., 82(17), 2008, 8828-8837.

[9] K.C. Huang, M.C. Lee, C.W. Wu, K.J. Huang, H.Y. Lei, and J.W. Cheng, Solution structure and neutralizing antibody binding studies of domain III of the dengue-2 virus envelope protein, Proteins, 70(3), 2008, 1116-1119.

[10] S.M. Lok, V. Kostyuchenko, G.E. Nybakken, H.A. Holdaway, A.J. Battisti, S. Sukupolvi-Petty, D. Sedlak, D.H. Fremont, P. R. Chipman, J.T. Roehrig, M.S. Diamond, R.J. Kuhn, M.G. Rossmann, Binding of a neutralizing antibody to dengue virus alters the arrangement of surface glycoproteins, Nat. Struct. Mol. Biol., 15(3), 2008, 312-317.

[11]S. Sukupolvi-Petty, S.K. Austin, W.E. Purtha, T. Oliphant, G.E. Nybakken, J.J. Schlesinger, J.T.Roehrig, G.D.
Gromowski, A.D. Barrett, D.H. Fremont, and M.S. Diamond, Type- and subcomplex-specific neutralizing antibodies against domain III of dengue virus type 2 envelope protein recognize adjacent epitopes, J. Virol., 81(23), 2007, 12816-12826.

[12] J.T. Roehrig, Antigenic structure of flavivirus proteins, $A d v$. Virus Res., 59, 2003, 141-175.

[13] W.D. Crill and J.T. Roehrig, Monoclonal antibodies that bind to domain III of dengue virus $\mathrm{E}$ glycoprotein are the most efficient blockers of virus adsorption to Vero cells, J. Virol., 75(16), 2001, 7769-7773.

[14] J.M. Reichert, Monoclonal antibodies in the clinic, Nat. Biotechnol., 19(9), 2001, 819-822.

[15] D. Schrama, R.A. Reisfeld, and J.C. Becker, Antibody targeted drugs as cancer therapeutics, Nat. Rev. Drug Discov., 5(2), 2006, 147-159.

[16] E. Waltz, Supreme Court boosts licensees in biotech patent battles, Nat. Biotechnol., 25(3), 2007, 264-265.

[17] P. Bork, Hundreds of ankyrin-like repeats in functionally diverse proteins: mobile modules that cross phyla horizontally? Proteins, 17(4), 1993, 363-374.

[18] S. Gorina and N.P. Pavletich, Structure of the p53 tumor suppressor bound to the ankyrin and SH3 domains of 53BP2, Science, 274(5289), 1996, 1001-1005.

[19] S.G. Sedgwick and S.J. Smerson, The ankyrin repeat: a diversity of interactions on a common structural framework, Trends Biochem. Sci., 24(8), 1999, 311-316.

[20] D. Steiner, P. Forrer, and A. Plückthun, Efficient selection of DARPins with sub-nanomolar affinities using SRP phage display, J. Mol. Biol., 382(5), 2008, 1211-1227.

[21] N. Sawatree, U. Agathe, V.L. Marie, K. Wannisa, S. Supachai, H. Saw-See, B. Pierre, M. Philippe, and T. Chatchai, Antiviral activity of recombinant ankyrin targeted to the capsid domain of HIV-1 Gag polyprotein, Retrovirology, 9(17), 2012, 695700.

[22] D.A. Case, T.A. Darden, T.E. Cheatham, III, C.L. Simmerling, J. Wang, R.E. Duke, R. Luo, R.C. Walker, W. Zhang, K.M. Merz, B. Roberts, S. Hayik, A. Roitberg, G. Seabra, J. Swails, A. W. Götz, I. Kolossváry, K. F. Wong, F. Paesani, J. Vanicek, R.M. Wolf, J. Liu, X. Wu, S.R. Brozell, T. Steinbrecher, H. Gohlke, Q. Cai, X. Ye, J. Wang, M.-J. Hsieh, G. Cui, D.R. Roe, D.H. Mathews, M.G. Seetin, R. Salomon-Ferrer, C. Sagui, V. Babin, T. Luchko, S. Gusarov, A. Kovalenko, P.A. Kollman, 2012, AMBER 12, University of California, San Francisco. 